\title{
La atención farmacéutica mejora los parámetros clínicos de las enfermedades crónicas
}

\author{
Pharmaceutical care improves clinical parameters of chronic diseases
}

\section{Objetivos}

Comparar la eficacia del manejo del tratamiento dirigido por el farmacéutico versus el cuidado habitual en pacientes con enfermedades crónicas.

\section{Fuentes de datos}

Se realizó una búsqueda en MEDLINE, Cochrane Library, CINAHL, y en resúmenes farmacéuticos internacionales desde 1995 hasta febrero de 2016. Adicionalmente, se revisaron las listas de referencias de revisiones sistemáticas y de los estudios incluidos.

\section{Selección de estudios}

Se incluyeron estudios de cualquier diseño que tuviesen un grupo control, que evaluaran pacientes adultos ambulatorios con factores de riesgo o enfermedades crónicas, los cuales recibieran alguna intervención en la cual el farmacéutico fuera responsable de un componente del cuidado del paciente, en alguna instalación de cuidado de salud de los EE.UU. Se excluyeron aquellos estudios que no involucraran intervenciones para prevenir o manejar una o más enfermedades crónicas, y los focalizados en clínicas de anticoagulación debido a que el manejo del farmacéutico es considerado estándar de cuidado en esos ámbitos.
Greer N y col. Ann Intern Med 2016;165:30-40.

Extracción de datos

Los datos fueron extraídos por un investigador y verificados por un segundo autor en forma independiente. Se agruparon los estudios según distintas patologías. El riesgo de sesgo (alto, moderado, bajo e insuficiente) fue evaluado por dos investigadores y la solidez de las pruebas fue determinada por consenso.

Los principales resultados de interés fueron los eventos clínicos, la mortalidad, la satisfacción del paciente, la utilización de servicios sanitarios (emergencia e internaciones) y la adherencia a la medicación. También se evaluaron los problemas relacionados con los medicamentos y los costos sanitarios.

\section{Resultados}

Se incluyeron un total de 63 estudios, entre los cuales había 40 ensayos clínicos controlados aleatorizados (ECCA), con un período máximo de seguimiento de 12 meses, realizados en pacientes ambulatorios con enfermedades crónicas de centros de salud de EE.UU. Los estudios comparaban la atención farmacéutica frente al cuidado usual de los pacientes en los tratamientos crónicos. Los principales resultados se detallan en la tabla 1.

Tabla 1. Resultados de los meta-análisis realizados para los desenlaces clínicos en distintas enfermedades crónicas o factores de riesgo cardiovascular

\begin{tabular}{|l|c|c|c|}
\hline \multicolumn{1}{c|}{ Enfermedad (desenlace) } & $\begin{array}{c}\text { Cuidado del farmacéutico } \\
\text { (eventos/total pacientes) }\end{array}$ & $\begin{array}{c}\text { Cuidado Usual } \\
\text { (eventos/total pacientes) }\end{array}$ & $\begin{array}{c}\text { Riesgo Relativo } \\
\text { (IC 95\%) }\end{array}$ \\
\hline Diabetes mellitus (HbA1c en meta*) & $121 / 308$ & $68 / 308$ & $1,83(1,44 \mathrm{a} 2.33)$ \\
\hline Dislipidemias (LDL-c en meta*) & $69 / 171$ & $50 / 163$ & $1,14(0,83 \mathrm{a} 2,40)$ \\
\hline Hipertensión arterial (PA en meta)) & $532 / 973$ & $383 / 1.014$ & $1,45(1,24 \mathrm{a} 1,70)$ \\
\hline
\end{tabular}

$\mathrm{HbA}$ : niveles de hemoglobina glicosilada sérica; LDL-c: cholesterol LDL; PA: presión arterial *Se definió como meta terapéutica un nivel de HbA1c menor o igual a 7\%. "Se definió como meta terapéutica un nivel de LDL-c menor de $100 \mathrm{mg} / \mathrm{dL}$ u otro valor. ${ }^{\complement}$ Se definieron como meta terapéutica los niveles de presión arterial menor de $140 / 90 \mathrm{mmHg}$

La mortalidad, la utilización de servicios sanitarios, la adherencia a la medicación y los costos tuvieron valores similares tanto en los pacientes con cuidado farmacéutico como en quienes recibieron cuidado usual. Con respecto a las dosis y la cantidad de medicamentos, se observó un incremento en los pacientes que recibieron seguimiento por parte de un farmacéutico. La evidencia para medir la satisfacción del paciente fue considerada como insuficiente y la notificación de los problemas relacionados con medicamentos fue limitada.

\section{Conclusión}

El manejo de las enfermedades crónicas a cargo del seguimiento de un farmacéutico estuvo asociada a efectos similares en comparación con el cuidado usual, sin embargo, se observaron buenos resultados en desenlaces intermedios como el control de la hemoglobina glicosilada, los niveles de colesterol LDL y la presión arterial.

Fuente de financiamiento/conflicto de interés de los autores: Administración de Salud de Veteranos de los EE.UU. Los autores declararon no tener conflictos de intereses.

\section{Comentario}

El farmacéutico es la fuente de información en relación al uso adecuado de los medicamentos y se ha observado que cuando brinda un seguimiento al paciente, se genera un aumento de la tasa de adherencia a los tratamientos y los hábitos saludables ${ }^{1}$. Son distintas las actividades que puede ejercer para ayudar al paciente, como evitar o detener las prescripciones que pueden asociarse con interacciones farmacológicas, ayudar a la adherencia de los tratamientos y orientar a los pacientes sobre hábitos cotidianos relacionados con la medicación. Si este trabajo se realiza en equipo con el profesional médico se pueden lograr mejores resultados. La revisión que hemos resumido pudo documentar beneficios en algunos desenlaces clínicos con la intervención del farmacéutico. A pesar de no haber suficiente evidencia sobre la satisfacción de los pacientes, dado que sólo 4 estudios evaluaron este resultado, esto no debería desestimar el potencial rol positivo del farmacéutico en el cuidado de los pacientes con enfermedades crónicas. Con respecto al grupo de pacientes con seguimiento farmacéutico que habían aumentado el número de medicamentos o la dosis de los mismos, es difícil evaluar si ese incremento es indicador de una mejor calidad de la atención.

\section{Conclusiones de los comentadores}

Si bien se demostró una mejora en los objetivos terapéuticos debido a la atención dirigida por el farmacéutico, se necesitan más investigaciones para determinar si el logro de los mismos se traduce en beneficios a largo plazo sobre desenlaces clínicos de los pacientes y los costos sanitarios.

Maria Jose Bellomo y Maite Ibargüen [ Senvicio de Farmacia, Hospital Italiano Buenos Aires; bellomo@ hospitalitaliano.org.ar, m.ibarguen@ hospitalitaliano.org.ar ] Bellomo MJ, Ibargüen M. La atención farmacéutica mejora los parámetros clínicos de las enfermedades crónicas. Evid Actual Pract Ambul 2018;21(3):75. Comentado de: Greer N y col. Pharmacist-Led Chronic Disease Management: A Systematic Review of Effectiveness and harms Compared With Usual Care. Ann Intern Med 2016;165:30-40. PMID: 271110098. 